\title{
In-flight estimation of the aerodynamics of tethered wings for airborne wind energy
}

\author{
Eduardo Schmidt*, Marcelo De Lellis*, Ramiro Saraiva*, Lorenzo Fagiano ${ }^{\dagger}$, and Alexandre Trofino* \\ *Department of Automation and Systems, Federal University of Santa Catarina, Florianópolis, SC, Brazil \\ ${ }^{\dagger}$ Dipartimento di Elettronica, Informazione e Bioingegneria, Politecnico di Milano, Milan, Italy
}

\begin{abstract}
A new filtering approach is presented, able to estimate online the aerodynamic characteristics of tethered wings used in airborne wind energy systems. The approach is based on the Extended Kalman Filter (EKF) and on a representative model of the flight dynamics. The filter is fed with measurements available at the ground station, namely the line angles and their rates, the traction force on the tether, and the wind speed and direction a few meters above ground. Convergence and accuracy of the estimates are first evaluated through simulations. Then, the approach is demonstrated using extensive experimental data, collected during field tests with two small-scale prototypes built independently by different research groups and four different flexible airfoils (power kites), of both ram-air type and of leading-edge-inflated type. The experimental results show that the algorithm can effectively estimate both the state and the aerodynamic parameters of the wings, as well as the wind speed and direction at the wing's altitude, and makes it possible to analyze for the first time the aerodynamics during highly dynamical tethered flight.
\end{abstract}

Index Terms-Airborne Wind Energy, Tethered wings, Aerodynamics, Nonlinear System Identification, Nonlinear Estimation, Extended Kalman Filter

\section{INTRODUCTION}

Airborne Wind Energy (AWE) systems harvest wind energy by exploiting the aerodynamic forces acting on tethered wings flying fast in crosswind conditions. Compared to the conventional wind turbines, this technology can reach higher altitudes, where the wind is stronger and more consistent, while allowing for a reduction in the construction and installation costs of the power plant. These features may provide good complementarity between AWE and established renewable energy technologies, thus contributing positively to energy security and sustainability. AWE systems consist of two major components, a ground station and an aircraft, which are mechanically and in some concepts even electrically connected by one or more tethers. A comprehensive survey of AWE technology can be found in [1], [2].

Many contributions in the AWE literature are concerned with control design and optimal system operation, see e.g.

Manuscript received June 20, 2018. This work was supported by $\mathrm{CNPq}$ under grants 480931/2013-5 and 406996/2013-0, and CAPES, Brazil. (Corresponding author: Eduardo Schmidt.)

E. Schmidt, M. De Lellis, R. Saraiva, and A. Trofino are with the Department of Automation and Systems, Federal University of Santa Catarina, 88040900 Florianópolis, SC, Brazil (e-mail: edufschmidt@gmail.com; mardelellis@gmail.com; ramiro.saraiva@gmail.com; alexandre.trofino@ufsc.br).

L. Fagiano is with the Dipartimento di Elettronica, Informazione e Bioingegneria, Politecnico di Milano, 20133 Milan, Italy (e-mail: lorenzo.fagiano@polimi.it).
[3], [4], [5], [6], [7], [8], [9], [10], [11]. In the majority of the approaches proposed, an accurate parametric model of the wing is assumed. In some cases, knowledge of the wind speed at the wing's position is assumed as well. However, such information is known only with a limited accuracy in practice. As an example, the aerodynamic characteristics of the wing, usually represented by curves that indicate how the lift and drag coefficients vary with the angle-of-attack, are difficult to characterize in ad-hoc experiments, especially for highly dynamical tethered flight conditions and soft or semi-rigid structures. Furthermore, even if the aerodynamic characteristics were initially known, they may change significantly during operation due to the effects of command inputs, environmental conditions (rain, snow, air humidity), or accumulated material wear.

This aspect raised the need for the implementation of additional mechanisms to estimate in real-time the model parameters and the wind conditions, based on measurements obtained from the system. These estimates can then be used to optimize the energy yield and control performance, to take high-level decisions such as take-off and landing, and to monitor the system conditions for the sake of fault detection and predictive maintenance. In [12], a model of the wind dynamics was used together with the flight dynamics model to estimate the model states with Kalman filters. A filtering scheme based on the Unscented Kalman Filter (UKF) was presented in [13]. Other works, such as [14], [15] presented setups for the online identification of the gain and transport delay between the steering input and the resulting change in direction of the wing. While the aerodynamic characteristics of the system are known to affect both these variables, the problem of determining this relationship and specifically computing the lift and drag coefficients has not been addressed so far in neither of these references. More recently, in [16], an estimation strategy inspired based on an EKF was presented, exploiting the perfect measurement technique to enforce the orthogonality between the estimated lift and apparent wind vectors, yielding the successful estimation of an augmented state vector comprising also the wind and the lift force vectors.

Building on the results presented in [16], this paper proposes and tests on extensive experimental data a new algorithm for the in-flight estimation of the aerodynamic lift and efficiency curves of tethered wings. Although it relies on the same minimal set of measurements and parameters used in [16], the filtering strategy hereafter presented is based on a dynamical model of the wing flight that significantly differs from that 
utilized in [16]. In spite of that, in can still be applied to any type of wing and has the potential to replace complex and expensive wind tunnel tests. In addition to the new model, the contributions of this paper are (a) the use of an orthogonality constraint within the filtering stage of the EKF. which significantly improves the convergence and stability of the estimator; and (b) the presentation and comparison of the aerodynamic curves estimated by the proposed algorithm from experimental data collected during a series of field tests with two small-scale AWE prototypes, developed independently by two research groups, and four airfoils of different types and sizes.

The paper is organized as follows: Section [II presents the employed model of the flight dynamics, which is later embedded into the EKF, as described in Section IIII Section IV] presents the experimental setups and procedures employed to assess the performance of the estimator. Finally, conclusions and future developments are included in Section $\mathrm{V}$.

\section{Notation and units}

We denote vectors by bold characters and their Euclidean norm by $\|.\| . x_{i}$ is $i$-th element of a discrete sequence $x$. In particular, for a continuous-time signal $x, x_{i}$ denotes its samples at time $i T$, where $T$ is the sample period. The components $(x, y, z)$ of a vector $\mathbf{r}$ are denoted by $r_{x}, r_{y}, r_{z}$. Specific parameters of the kite and tether are indicated by the subscript $k$ and $t$, respectively.

\section{FLIGHT DYNAMICS MODEL}

Consider a wing linked to a ground station by one or more tethers, and operating as an AWE system with either groundbased generation (lift power mode) or airborne generation (drag power mode), see e.g. [2] and Fig. 1 for one of the experimental setups employed in this work. As a fair compromise between simplicity and accuracy for filtering purposes, the airborne system is modeled as two point-masses. The first one corresponds to the kite, with mass $m_{k}$, possibly also comprising a control pod. The second point-mass corresponds to tethers, which are assumed to be perfectly taut, with mass $m_{t}$ placed midway between the ground anchoring point and the kite point-mass position. These are usual assumptions in the literature on AWE systems. Knowing that $n_{t}$ is the number of tethers, and $d, L$ and $\mu_{t}$ are the tether diameter, length and volumetric density, respectively, the total tether mass is given by

$$
m_{t}=(1 / 4) n_{t} \pi d^{2} L \mu_{t} .
$$

Besides the weight, the tethers also suffer an aerodynamic drag force. Later on, in order to assemble the equations of motion, these two forces will be translated to the kite pointmass position by keeping constant their produced momen $\square$. yielding a single equivalent point with mass $m$ concentrated

\footnotetext{
${ }^{1}$ If the tether is thought of as a straight rod, any force with a component perpendicular to the rod axis can be translated to the rod upper end (the kite) by keeping constant the torque produced by the force at the position where it is applied.
}

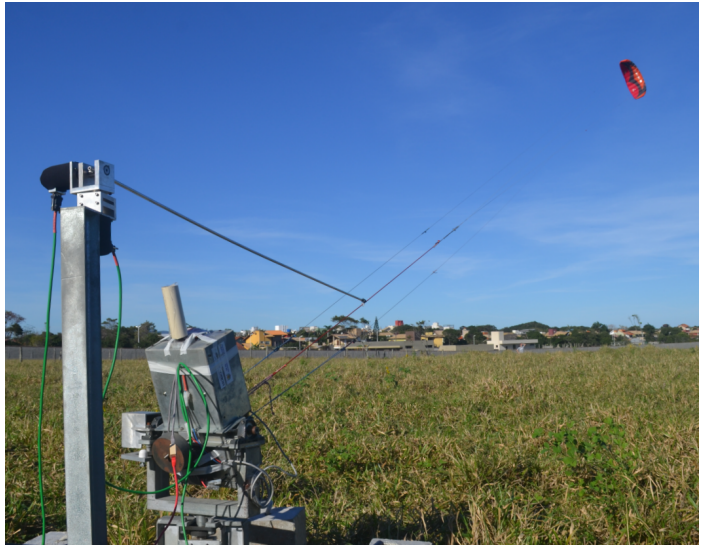

(a) Overview of the prototype in operation.

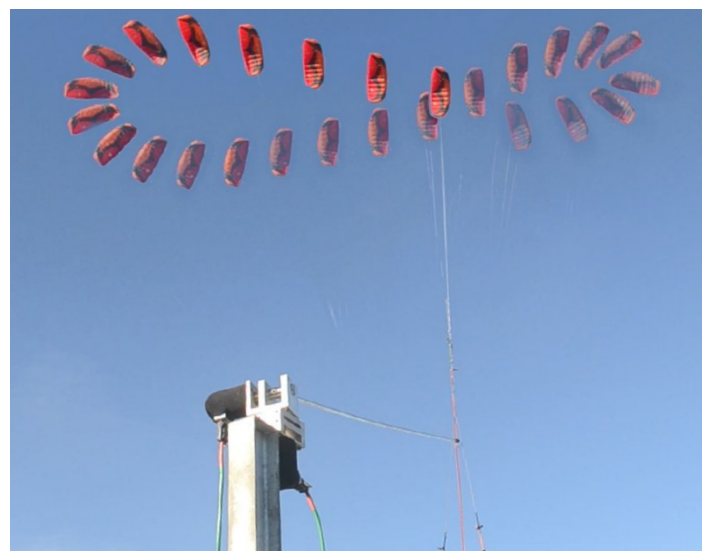

(b) Time-lapse image of the flight trajectory (one orbit).

Fig. 1: Small-scale AWE prototype of the UFSCkite group with ground-based actuation for study of flight aspects.

at the wing's position. Since the center of mass of the pointmass wing coincides with its aerodynamic center, the forces of lift $\mathbf{F}_{L}$, equivalent drag $\mathbf{F}_{D}$, equivalent weight $\mathbf{F}_{G}$, tether traction $\mathbf{F}_{T}$ and apparent forces $\mathbf{F}_{P}$ (Coriolis and centripetal) all act upon $m$, as depicted in Figure 2. Note that the attribute "equivalent" refers to the effects of the tether drag and weight combined with those of the kite.

Before deriving the equations of motion, it is important to highlight that the usual assumption in models such as those proposed by [4], [17] is that the wing evolves on a sphere centered at the lower attachment point of the tether - a constraint that is naturally embedded in the model when using spherical coordinates. The wing orientation is often represented by Euler angles, or quaternions. In [18], a modeling approach that yields singularity-free differential equations in natural (Cartesian) coordinates is presented. This paper follows a similar approach, by accounting for the multi-body nature of the system, and by linking these bodies through algebraic constraints incorporated into the equations of motion in a Lagrangian framework.

The suspended structure in Figure 2 is exposed to a wind vector $\mathbf{w}_{n}$ with components only in the horizontal plane, i.e. in the directions of $\mathbf{x}_{0}$ and $\mathbf{y}_{0}$ only. The potential energy of the system is defined as $V(\mathbf{q})$, and its kinetic energy as $T(\mathbf{q}, \dot{\mathbf{q}})$, 


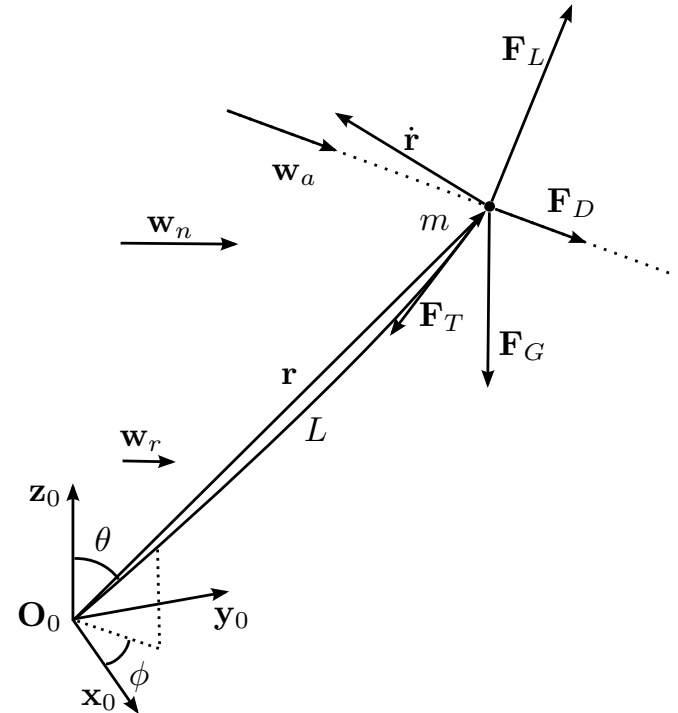

Fig. 2: AWE system represented by an equivalent pointmass, located at the aerodynamic/mass center and subject to a number of forces, including aerodynamic ones.

where $\mathbf{q}=\left(q_{1}, q_{2}, \ldots, q_{n}\right)$ are the generalized coordinates In order to work with Cartesian coordinates, the generalized coordinates vector $\mathbf{q}$ is defined as $\mathbf{q}=\mathbf{r}=\left[r_{x}, r_{y}, r_{z}\right]^{T}$, which is the position of the kite point-mass $m_{k}$. Knowing that the tether point-mass $m_{t}$ is located at $(1 / 2) \mathbf{r}$, the expressions of the system's potential and kinetic energy are, respectively,

$$
\begin{aligned}
V(\mathbf{r}) & =\left(m_{k}+\frac{1}{2} m_{t}\right) g r_{z} \\
T(\mathbf{r}, \dot{\mathbf{r}}) & =\frac{1}{2}\left(m_{k}+\frac{1}{4} m_{t}\right) \dot{\mathbf{r}}^{T} \dot{\mathbf{r}}=(1 / 2) m \dot{\mathbf{r}}^{T} \dot{\mathbf{r}},
\end{aligned}
$$

where $g$ is the acceleration of gravity, $m$ is the equivalent mass, and $\dot{\mathbf{r}}$ is the wing velocity with respect to the reference frame. Based on these equations, the system Lagrangian can be built as

$$
\mathcal{L}(\mathbf{r}, \dot{\mathbf{r}}, \nu)=T(\mathbf{r}, \dot{\mathbf{r}})-V(\mathbf{r})-\nu^{T} c(\mathbf{r}),
$$

where $\nu$ is a vector of Lagrange multipliers, each corresponding to a given constraint in the vector of constraints $c(\mathbf{r})$. In this specific model, the only constraint is that the distance from the kite to the ground station is equal to the tether length, i.e. $\|\mathbf{r}\|=L$. As discussed by [18], in a general scenario with variable tether length, the constraint and its time-derivatives can be expressed as

$$
\begin{aligned}
c(\mathbf{r}) & =(1 / 2)\left(\mathbf{r}^{T} \mathbf{r}-L^{2}\right)=0 \\
\dot{c}(\mathbf{r}, \dot{\mathbf{r}}) & =\mathbf{r}^{T} \dot{\mathbf{r}}-L \dot{L}=0 \\
\ddot{c}(\mathbf{r}, \dot{\mathbf{r}}, \ddot{\mathbf{r}}) & =\mathbf{r}^{T} \ddot{\mathbf{r}}+\dot{\mathbf{r}}^{T} \dot{\mathbf{r}}-L \ddot{L}-\dot{L}^{2}=0 .
\end{aligned}
$$

Denoting the sum of all the external forces acting upon the system by $\sum \mathbf{F}_{\text {ext }}$, the equations of motion can be obtained by substitution of (3) into the Euler-Lagrange equations

$$
\frac{\partial \mathcal{L}(\mathbf{r}, \dot{\mathbf{r}}, \nu)}{\partial r_{j}}-\frac{d}{d t} \frac{\partial \mathcal{L}(\mathbf{r}, \dot{\mathbf{r}}, \nu)}{\partial \dot{r}_{j}}+\sum \mathbf{F}_{e x t}=0,
$$

evaluated for each $j$-th coordinate of $\mathbf{r}$. Considering also the constraint in (4), the following set of dynamic equations can be obtained:

$$
\underbrace{\left[\begin{array}{cc}
m \mathbf{I}_{3} & \mathbf{r} \\
\mathbf{r}^{T} & 0
\end{array}\right]}_{\mathbf{M}}\left[\begin{array}{c}
\ddot{\mathbf{r}} \\
\nu
\end{array}\right]=\left[\begin{array}{c}
\sum \mathbf{F}_{\text {ext }}+\left(m_{k}+\frac{1}{2} m_{t}\right) \mathbf{g} \\
-\dot{\mathbf{r}}^{T} \dot{\mathbf{r}}+\dot{L}^{2}+L \ddot{L}
\end{array}\right],
$$

where $\mathbf{I}_{3}$ is the identity matrix of size 3 , and $\mathbf{g}=[0,0,-g]^{T}$ is Earth's gravity vector. Hence, given the system parameters $\left(m_{k}, m_{t}\right.$ and $g$ ), the sum of external forces $\sum \mathbf{F}_{\text {ext }}$, and the (possibly controlled) inputs of tether length $L$, reel-out speed $\dot{L}$ and acceleration $\ddot{L}$, the solution vector $[\ddot{\mathbf{r}}, \nu]^{T}$ can be computed as long as $\mathbf{M}$ is invertible.

Observe that the quantities in both sides of (6) are forces. As pointed out in [18], since $\nu$ corresponds to the constraint on the wing flight due to the tether, the force that arises from this constraint, i.e. the tether traction force $\mathbf{F}_{T}$ can be directly computed as

$$
\mathbf{F}_{T}=\mathbf{r} \nu
$$

The effect of the apparent forces $\mathbf{F}_{P}$ are implicitly considered in the model through the second row of (6). On the other hand, the weight vector is explicit, defined as $\mathbf{F}_{G}=\left(m+\frac{1}{2} m_{t}\right) \mathbf{g}$.

The external forces acting upon the system are the aerodynamic forces of lift and drag, which result from the interaction of the airfoil and tether with the apparent wind, defined as:

$$
\mathbf{w}_{a}=\mathbf{w}_{n}-\dot{\mathbf{r}} .
$$

The lift force $\mathbf{F}_{L}$ lies in the plane perpendicular to the apparent wind $\mathbf{w}_{a}$, but its instantaneous direction is a priori unknown, being described by the unit vector $\mathbf{z}_{L}$. As opposed to what happens to the lift force, the direction and orientation of the drag force are solely determined by the apparent wind. The drag forces on tether and the airfoil are combined into a single drag force $\mathbf{F}_{D}$, whose direction and orientation coincide with those of $\mathbf{w}_{a}$. Knowing that $\rho$ is the air density, $A$ is the wing projected area, and $C_{L}$ and $C_{D}$ are the lift and equivalent drag coefficients, respectively, the aerodynamic forces are defined as

$$
\begin{aligned}
& \mathbf{F}_{L}=(1 / 2) \rho A C_{L} w_{a}^{2} \mathbf{z}_{L} \\
& \mathbf{F}_{D}=(1 / 2) \rho A C_{D} w_{a}^{2}\left(\mathbf{w}_{a} / w_{a}\right) .
\end{aligned}
$$

According to [19], the equivalent drag coefficient can be computed as

$$
C_{D}=C_{D k}+\frac{n_{t} C_{D t} L d \cos (\Delta \alpha)}{4 A},
$$

where $C_{D k}$ is the drag coefficient of the kite alone, and $\Delta \alpha$ is the angle between the apparent wind $\mathbf{w}_{a}$ and the tangent plane at $\mathbf{r}$ of a sphere of radius $\|\mathbf{r}\|$, defined as

$$
\Delta \alpha=\arcsin \left(\frac{\mathbf{w}_{a}}{\left\|\mathbf{w}_{a}\right\|} \cdot \frac{\mathbf{r}}{\|\mathbf{r}\|}\right) .
$$

Furthermore, assuming that the angle $\alpha_{0}$ between the wing chord line and the tangent plane at $\mathbf{r}$ of a sphere of radius 
$\|\mathbf{r}\|$ is known, one can compute the airfoil angle of attack as $\alpha=\alpha_{0}+\Delta \alpha$. The aerodynamic coefficients are strongly dependent on $\alpha$, as will be seen in Section IV

Flight actuation takes place by means of the control vector $\mathbf{u}=\left[u_{s}, u_{p}\right]^{T}$ comprising a steering and a pitch/de-power command, $u_{s}$ and $u_{p}$, respectively. Regardless of the specific type of wing, as a general rule, the steering input causes the lift force to rotate in the plane perpendicular to the apparent wind vector at an angular velocity $\omega_{l}$. As for the second input, it either directly changes the angle of attack by pitching the aircraft or it alters the airfoil shape and the corresponding curves of the lift and drag coefficients. In both cases, the input $u_{p}$ causes the magnitude of the aerodynamic forces to change. Figure 3 illustrates this mechanism of flight actuation, where $k_{s}$ and $k_{p}$ are positive constants that depend on specific design parameters.

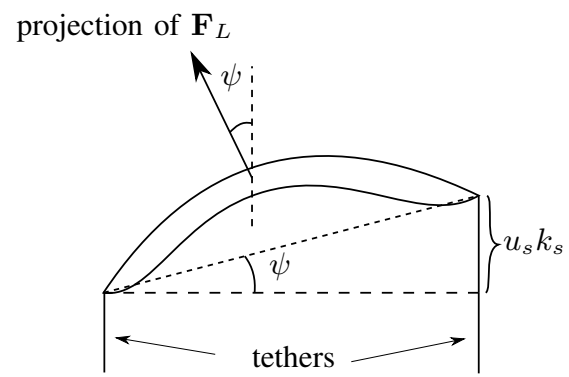

(a) Front view: roll angle $\psi$ due to steering input $u_{s}$.

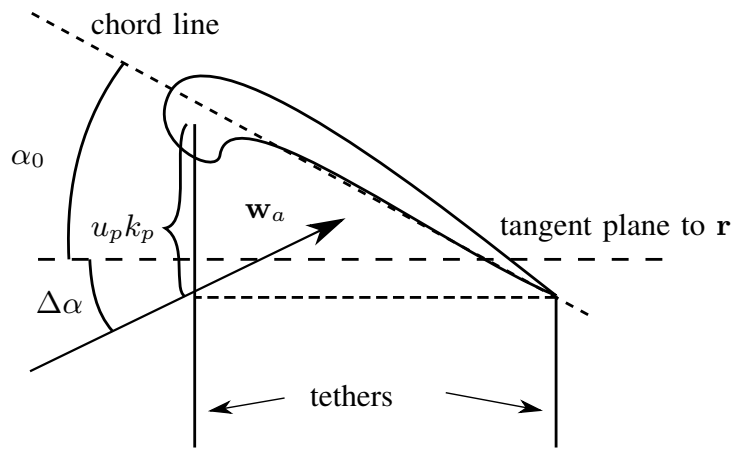

(b) Side view: pitch angle $\alpha_{0}$ due to depower input $u_{p}$.

Fig. 3: Example of flight actuation in the case of a 3-tethered kite with roll and pitch angle maneuverability.

It is worth emphasizing that the equations of motion presented in (6) also include the tether traction and apparent forces, in contrast to what was presented in [16], whose modeling completely neglected these variables. As a result, more accurate filtering results have been achieved.

\section{FILTER DESIGN}

The presented model equations can be employed in conjunction with an observation model in an EKF framework for the purpose of state and parameter estimation. To this end, a state vector $\mathbf{x}$ is proposed, which contains the position $\mathbf{r}$, the velocity $\dot{\mathbf{r}}$, and the acceleration $\ddot{\mathbf{r}}$ of the equivalent pointmass. These states are followed by the Lagrange multiplier $\nu$ associated to the tether constraint, the nominal wind $\mathbf{w}_{n}$, the lift force $\mathbf{F}_{L}$, the magnitude of the equivalent drag force $F_{D}$, and by a scalar gain $c_{u}$ describing a linear relationship between the steering input and the angular rate at which $\mathbf{F}_{L}$ rotates around the apparent wind:

$$
\mathbf{x}=\left[\mathbf{r}^{T}, \dot{\mathbf{r}}^{T}, \ddot{\mathbf{r}}^{T}, \nu, \mathbf{w}_{n}^{T}, \mathbf{F}_{L}^{T}, F_{D}, c_{u}\right]^{T} .
$$

The evolution of the filter states is ruled by the flight dynamics discussed in the previous section, discretized with a finite differences approximation:

$$
\begin{aligned}
\mathbf{r}_{i+1} & =\mathbf{r}_{i}+\dot{\mathbf{r}}_{i} T_{s} \\
\dot{\mathbf{r}}_{i+1} & =\dot{\mathbf{r}}_{i}+\ddot{\mathbf{r}}_{i} T_{s} \\
{\left[\begin{array}{c}
\ddot{\mathbf{r}}_{i+1} \\
\nu_{i+1}
\end{array}\right] } & =\left[\begin{array}{cc}
m \mathbf{I}_{3} & \mathbf{r}_{i} \\
\mathbf{r}_{i}^{T} & 0
\end{array}\right]^{-1}\left[\begin{array}{c}
\mathbf{F}_{L i}+\mathbf{F}_{D i}+\left(m_{k}+\frac{1}{2} m_{t}\right) \mathbf{g} \\
-\dot{\mathbf{r}}_{i}^{T} \dot{\mathbf{r}}_{i}+\dot{L}^{2}+L \dot{L}
\end{array}\right] \\
\mathbf{w}_{n i+1} & =\mathbf{w}_{n i} \\
\mathbf{F}_{L i+1} & =\mathcal{R}\left(\mathbf{F}_{L i}, \mathbf{w}_{a i}, c_{u i} u_{s i} T_{s}\right) \\
F_{D i+1} & =F_{D i} \\
c_{u i+1} & =c_{u i},
\end{aligned}
$$

where $\mathcal{R}(\mathbf{u}, \mathbf{v}, \psi)$ is a function representing the counterclockwise rotation of $\mathbf{u}$ around $\mathbf{v}$ by an angle $\psi$, and $T_{s}$ is the sampling time. Observe from 13 that the steering input, $u_{s}$, directly affects the aerodynamic lift force. Although it does not explicitly appears in the equations, the de-power input $u_{p}$ indirectly affects the aerodynamic forces through the coefficients $C_{L}$ and $C_{D}$.

Independently of their design, in AWE systems a minimal set of measured variables is always available. These include the aircraft position $\mathbf{r}$ and velocity $\dot{\mathbf{r}}$ with respect to the ground station, the magnitude $F_{t}$ of the traction force on the main tether, and the wind speed $w_{r}$ and direction $\phi_{r}$ at a reference altitude $z_{r}$. These quantities were then translated into an observation vector given by

$$
\mathbf{y}=\left[\mathbf{r}^{T}, \dot{\mathbf{r}}^{T}, w_{r}, \phi_{r}, F_{T}, \delta\right]^{T},
$$

In the experiments considered in this paper, in both prototypes the wing position $\mathbf{r}$ and velocity $\dot{\mathbf{r}}$ were computed according to the equations

$$
\mathbf{r}=\left[\begin{array}{c}
L \sin (\theta) \cos (\phi) \\
L \sin (\theta) \sin (\phi) \\
L \cos (\theta)
\end{array}\right]
$$

and

$\dot{\mathbf{r}}=\left[\begin{array}{c}\dot{L} \sin (\theta) \cos (\phi)+L(\dot{\theta} \cos (\theta) \cos (\phi)-\dot{(} \phi) \sin (\theta) \sin (\phi) \\ \dot{L} \sin (\theta) \sin (\phi)+L(\dot{\theta} \cos (\theta) \sin (\phi)+\dot{(} \phi) \sin (\theta) \cos (\phi) \\ \dot{L} \cos (\theta)-L \dot{\theta} \sin (\theta)\end{array}\right]$,

from the tether azimuth and polar angles $(\phi$ and $\theta)$ and their rates of change, as measured by two rotary encoders. The magnitude of the tether traction force $F_{T}$, which is related to the filter states according to Equation 7, was measured by a load cell at the ground station. Finally, the wind speed $w_{r}$ and direction $\phi_{r}$ at a reference altitude $z_{r}$ (roughly $2 \mathrm{~m}$ from the 
ground) were measured with an off-the-shelf cup anemometer. The latter variables are related to the nominal wind at the kite altitude by an assumed logarithmic wind shear profile [20], given by the equations

$$
\begin{aligned}
& w_{r}=\frac{\log \left(z_{r} / z_{0}\right)}{\log \left(z / z_{0}\right)}\left\|\mathbf{w}_{n}\right\| \\
& \phi_{r}=\arctan \left(w_{n y} / w_{n x}\right),
\end{aligned}
$$

where $\mathbf{w}_{n}=\left[w_{n x}, w_{n y}, 0\right]^{T}$ is the nominal wind at $\mathbf{r}, \log (\cdot)$ is the natural logarithm, and $z_{0}$ is the surface roughness coefficient.

Regarding the observation $\delta$, it does not model any physical quantity. It is, in fact, a made-up variable representing the inner product between the lift vector and the apparent wind:

$$
\delta=\mathbf{F}_{l}^{T} \mathbf{w}_{a}
$$

and is included in the filtering as a way to enforce the orthogonality constraint between these two variables $(\delta=0)$, according to the perfect measurement technique presented in [21]. In practice, this means that the value of $\delta$ is always zero, and it is considered an extremely reliable observation, which in a Kalman filtering scenario implies that its associated covariance is negligible. Based on a thorough literature review, the inclusion of such an orthogonality constraint in the form of a measurement is a novel practice in AWE Note that the approach is particularly favored in this formulation given that both the lift force and the apparent wind can be easily obtained from the filter state vector.

Finally, from the filter outputs, the equivalent aerodynamic efficiency $E$ is computed by applying its definition:

$$
E=\frac{\left\|\mathbf{F}_{L}\right\|}{F_{D}} .
$$

\section{RESUlts}

A discrete-time EKF was implemented as in [22], with state and observation vectors given by (12) and (14), respectively. All derivatives required to propagate the state and the covariance matrices in the Kalman algorithm were numerically computed using forward finite differences with a step size $\epsilon=1 \times 10^{-5}$. The performance of the filter was first assessed within a simulation environment built based on a well-studied model. The goal of this initial validation phase was to verify how the estimated variables yielded by our algorithm adhered to their true values, which were readily available within the simulation environment. The filter was then evaluated on data from field tests with two different prototypes, allowing us unveil the aerodynamic properties of four different flexible wings during tethered flight.

\section{A. Simulation results}

The simulation results were obtained with the widely accepted tethered kite model presented in [4], with the modifications proposed by [19]. In this model, the kite is considered as a single point-mass with a known projected area. The steering input causes the kite to roll around its longitudinal axis, similarly to an airplane when its ailerons are actuated. In terms of control strategy, the simulated tethered wing was driven by means of a two-loop control topology similar to that described in [23], with gains conservatively adjusted to provide an over damped closed-loop response in a turbulencefree environment, resulting in a lemniscate-shaped trajectory.

The simulation parameters were chosen in order to match as closely as possible the foil (ram-air) kite used by the UFSCkit $^{2}$ group in its field experiments. The most relevant model parameters are summarized in Table I. The aerodynamic curves of the simulated kite are the ones considered in [24].

\begin{tabular}{|l|l|c|c|}
\hline \multicolumn{2}{|c|}{ Parameter } & \multicolumn{2}{c|}{ Value } \\
\hline Symbol & Description & Simulation & Prototype \\
\hline$A$ & Airfoil area & $3 \mathrm{~m}^{2}$ & $3 \mathrm{~m}^{2}$ \\
\hline & Airfoil aspect ratio & 3 & 3 \\
\hline$m$ & Airfoil mass & $0.5 \mathrm{~kg}$ & $0.5 \mathrm{~kg}$ \\
\hline$\alpha_{0}$ & Base angle of attack & $6.8^{\circ}$ & $($ unknown) \\
\hline$d_{t}$ & Tether diameter & $2 \mathrm{~mm}$ & $2 \mathrm{~mm}$ \\
\hline$\mu_{t}$ & Tether density & $970 \mathrm{~kg} / \mathrm{m}^{3}$ & $970 \mathrm{~kg} / \mathrm{m}^{3}$ \\
\hline$C_{d t}$ & Tether drag coefficient & 1.2 & $($ unknown) \\
\hline$L$ & Tether length (fixed) & $46 \mathrm{~m}$ & $46 \mathrm{~m}$ \\
\hline$z_{0}$ & Roughness coefficient & $0.01 \mathrm{~m}$ & $($ unknown) \\
\hline$z_{r}$ & Reference height & $2.5 \mathrm{~m}$ & $2.5 \mathrm{~m}$ \\
\hline
\end{tabular}

TABLE I: Parameters of the AWE system simulation model from [4], and of the UFSCkite prototype used in the corresponding field experiment.

At first, the filter convergence was verified in an ideal scenario, with no measurement noise and turbulence-free wind. The filter managed to keep its good performance even after noise was applied to the measurements. More specifically, regarding the components of the lift force vector, Figure $4 \mathrm{~b}$ presents a comparison between the actual value, obtained from the simulation, and the estimated value in the ideal scenario. In Figure $4 \mathrm{c}$ the same comparison is presented for the magnitude of the equivalent drag force. In both cases, the filter is able to track with reasonable accuracy the true values of the target variables.

After having validated the tracking performance of the filter, the latter was employed to study the aerodynamic characteristics of the wing. The original goal was to reconstruct the curves of $C_{L}(\alpha)$ and $C_{D k}(\alpha)$ used in the simulation. The results are shown in Figure 5, attesting that the proposed filter could indeed be used for reconstructing the actual aerodynamic curves, shown with red solid lines.

\section{B. Field test results with the UFSCkite prototype}

The filtering strategy was also evaluated on data from a field experiment with the prototype of the UFSCkite group. This system, shown in Figure 1 and whose parameters are summarized in Table I consists of a small foil kite tethered to a ground actuation structure. The prototype is equipped with rotary encoders for measuring the tether angles (azimuth and elevation), and their rates, from which the wing position and velocity vectors can be computed. A cup anemometer installed

${ }^{2}$ UFSCkite - Research group on tethered airfoils for wind power, hosted at the Federal University of Santa Catarina, in Florianópolis, Brazil. More info: trofino.das.ufsc.br/ufsckite 


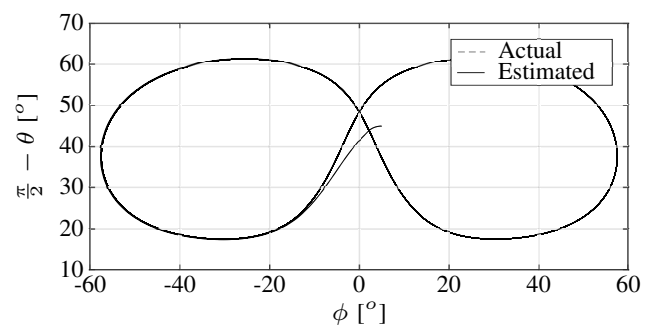

(a) Trajectory.

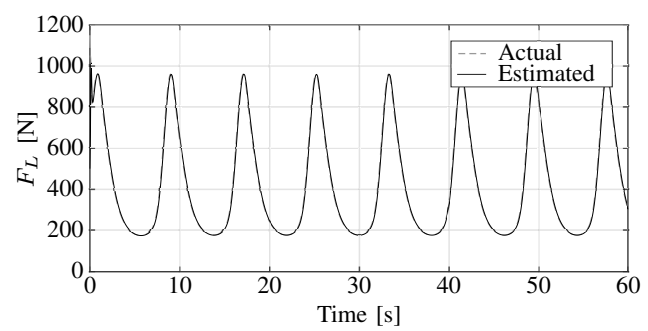

(b) Lift force.

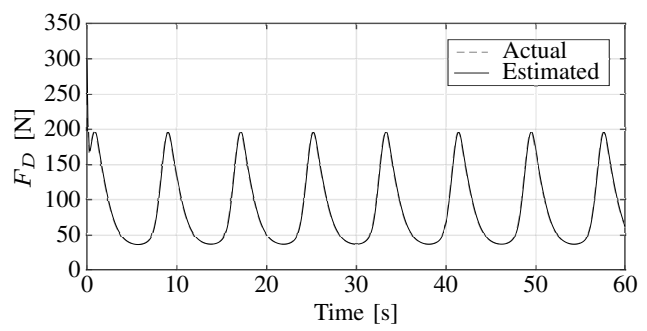

(c) Equivalent drag force.

Fig. 4: Comparison of the estimated and actual kite trajectory as well as of the aerodynamic forces magnitudes in the simulation environment with no wind turbulence nor measurement noise.

at an approximate height of $2.5 \mathrm{~m}$ measures the wind speed and direction, while a load cell measures the traction force.

The adopted control scheme comprises two cascaded feedback loops which resemble the structure presented in [15], [19]. The outer loop is responsible for generating course angle references using the algorithm proposed in [10], whereas the inner loop is responsible for tracking the desired course angle through a simple proportional controller.

In Figure 6, estimation results obtained during a field experiment are presented. Observe in Figure 6c that the lift force magnitude is quite low, averaging $300 \mathrm{~N}$, probably due to both the kite size $\left(3 \mathrm{~m}^{2}\right)$ and the wind conditions, which are shown in Figure 6b. As a consequence, the average traction force is not large enough to keep the tethers permanently taut. Besides introducing a delay in the kite position and speed measurements, the tether sag also represents an important source of modeling error for the filter. Note also that, in this experimental scenario, the tuning of the EKF had to be very conservative, mainly because of the noisy characteristics of the signal measured by the load cell.

As opposed to what happens in the simulation environment, the true values of the estimated variables are unknown, hence

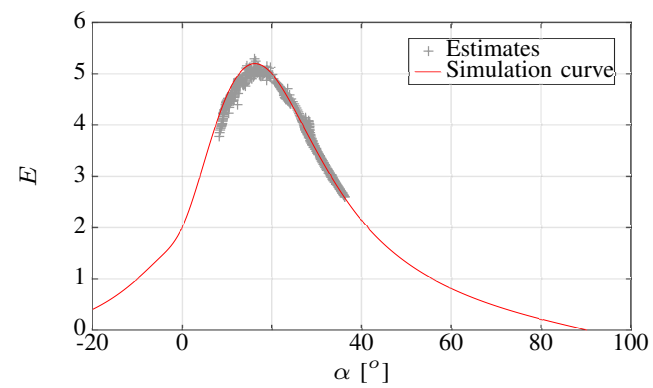

(a) Equivalent aerodynamic efficiency.

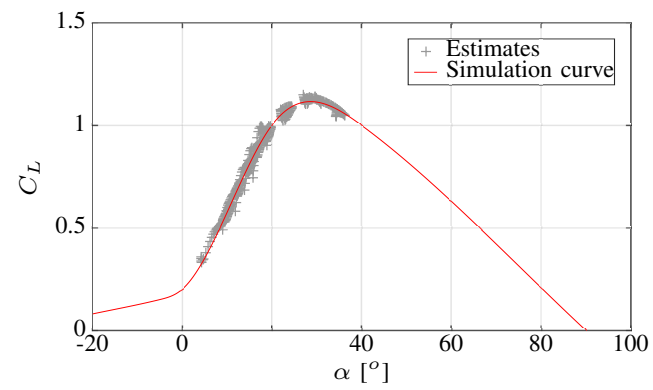

(b) Lift coefficient

Fig. 5: Estimates of the airfoil aerodynamic coefficients during several simulations, each carried out with a different base angle-of-attack $\alpha_{0}$, resulting in different intervals of $\alpha$.

not available for the sake of accuaracy validation in the field experiments. Thus, to assess the quality of the estimation results, a theoretical model of the tethered wing in dynamic equilibrium was utilized. This model, presented in detail in [9], assumes that all variables associated to the operation of the kite, such as the nominal wind and the aerodynamic coefficients, have negligible variation. It is worth stressing that this also includes the tether length, the angle-of-attack, and the polar and azimuth angles. If this assumption is valid, these variables can then be plugged into the equation.

$$
F_{T}=\frac{1}{2} \rho A C_{L} E^{2}\left(1+1 / E^{2}\right)^{3 / 2}\left(w_{n} \sin \theta \cos \phi\right)^{2}
$$

to compute the average (expected) value of the traction force.

For each orbit, the average values of the estimated polar angle $\theta$ and azimuth angle $\phi$ (obtained from the estimated kite position $\mathbf{r}$ ), the nominal wind $w_{n}$, the equivalent efficiency $E$, the lift coefficient $C_{L}$, and the known constant values of the air density $\rho$ and kite area $A$ were computed. These values were applied to (20), yielding theoretical values of average traction force $F_{T}$, which were then compared to the average of the measured traction force in each orbit. The results can be seen in Figure 7. The average measured values of $F_{T}$ are well centered at the expected theoretical values represented by the solid line, although with a considerable dispersion. This is probably due to the relatively large oscillation of the variables associated to model (20) induced by the low values of $F_{T}$, which also gives rise to a considerable tether sag. These combined effects reduce the validitiy of the modeling assumptions. 


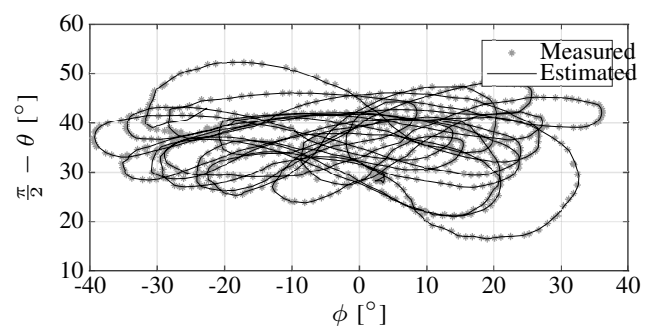

(a) Flight trajectory in the elevation-azimuth plane.

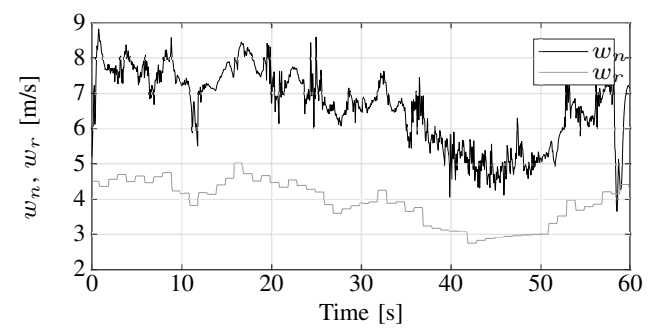

(b) Estimated wind at kite (black line) and reference (gray) height.

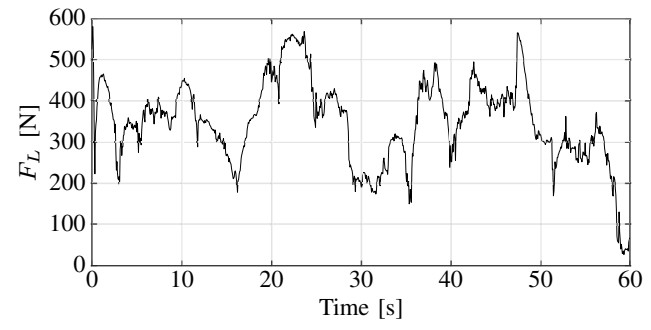

(c) Magnitude of the estimated lift force.

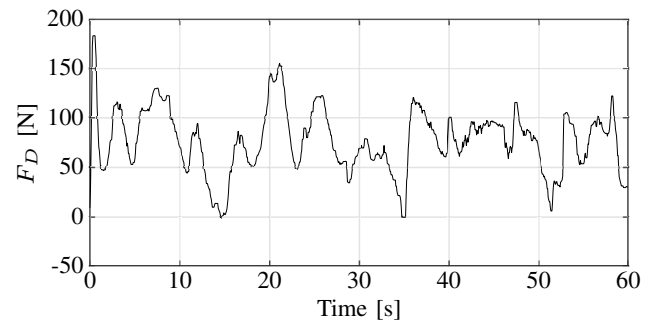

(d) Magnitude of the estimated equivalent drag force.

Fig. 6: Estimation results with the $3 \mathrm{~m}^{2}$ kite of the UFSCkite prototype during a field experiment.

The experimental data also allowed to estimate, to a limited extent, the aerodynamic properties of the UFSCkite prototype. In Figure $8 \mathrm{a}$ the crosses represent the lift coefficient $C_{L}$ estimated at each execution period of the filter as a function of the dynamic share of the angle of attack $\Delta \alpha$, along with a fitted curve using a $3^{\text {rd }}$ degree polynomial. As it happens with other $\Delta \alpha \times C_{L}$ and $\Delta \alpha \times E$ scatter plots in this document, the darker markers in Figure 8 are obtained by applying a moving median-based outlier rejection procedure to the original estimates yielded by the filter, which are, in turn, indicated in a lighter color. Usually the aerodynamic coefficients of lift, drag and efficiency are represented as a function of the (total) angle of attack $\alpha=\alpha_{0}+\Delta \alpha$ but, in this paper, $\Delta \alpha$ is utilized as the function domain since the

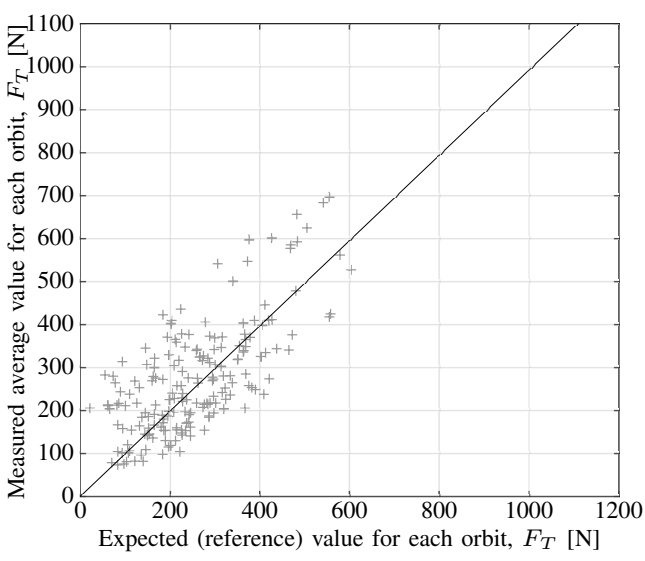

Fig. 7: Validation of the tether force estimates obtained in field tests with the UFSCkite prototype and a $3 \mathrm{~m}^{2}$ ram-air kite. Dots: experimental data. Solid line: theoretical value.

base angle of attack $\alpha_{0}$ is not precisely known. Observe in Figure 8 that $\Delta \alpha$ had a variation of approximately $20^{\circ}$, and the dispersion of each estimate around the fitted curve was quite large, which can be due to the factors already discussed.

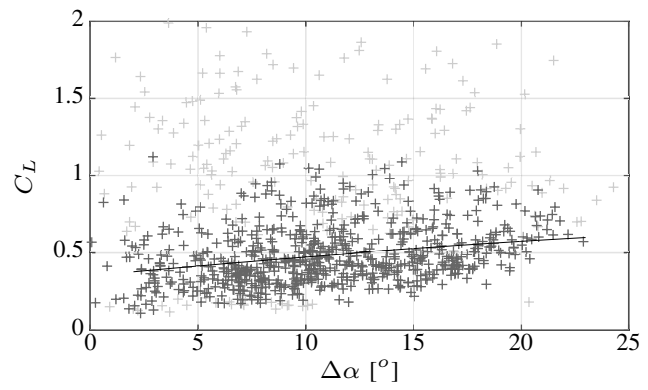

(a) Lift curve.

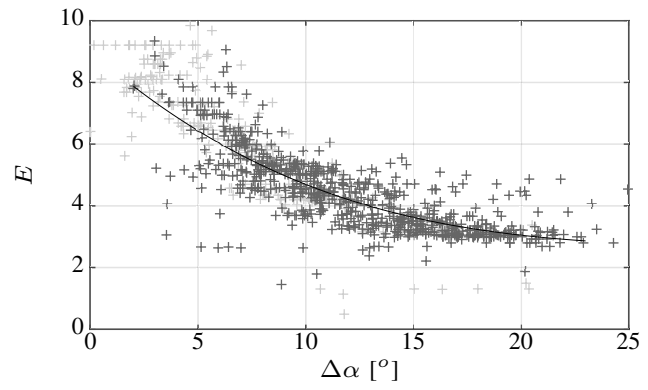

(b) Efficiency curve.

Fig. 8: Identification of the aerodynamic lift and efficiency of the UFSCkite prototype with a $3 \mathrm{~m}^{2}$ foil (ram-air) kite.

Scatter plots of the equivalent aerodynamic lift and efficiency are shown in Figure 8 along with fitted curves using a $3^{\text {rd }}$ degree polynomial. Observe how the dispersion of the $E$ estimates is now clearly lower than in the case of the $C_{L}$ estimation. This is probably because in the computation of $E$ with (19) and (9), the apparent wind $w_{a}$ is canceled out and therewith any sources of noise or uncertainty that this 
estimated quantity contains. Also note in Figure $8 \mathrm{~b}$ that the kite operates in a region where $E(\Delta \alpha)$ is decreasing, probably after a peak on the left side, whereas in Figure $8 \mathrm{a} C_{L}(\Delta \alpha)$ increases towards a probable peak on the right side. According to a study in [19] based on the aerodynamic curves from [24], the optimal base angle of attack $\alpha_{0}$ for reel-out power maximization should be such that the average angle of attack $\alpha$ lies between the peaks of $E(\alpha)$ and $C_{L}(\alpha)$. That study suggests that, during the experiment corresponding to Figure $8 \mathrm{~b}$, the kite was flying with an angle of attack inside an interval in which the optimum value for maximum power is expected to be. Hence, although $\alpha_{0}$ was unknown, it was probably well adjusted (we note that $\alpha_{0}$ can be tuned by adjusting the kite bridles).

\section{Field test results with the UCSB prototype}

The second considered prototype was built at the University of California, in Santa Barbara, USA, and is shown in Figure 9

This prototype operates with $30 \mathrm{~m}$ of constant tether length. Its geographical location in North-East-Down (NED) coordinates as well as the angle with respect to the geographical North are obtained through a GPS and three magnetometers. Moreover, the ground unit is equipped with a line angle measurement system (shown in Figure 9b), which provides a direct measurement of the angles between the main line connecting the wing to the ground unit and the axes of the inertial reference system fixed with the latter. This sensor was developed and built ad-hoc for this application and employs two incremental encoders. In some tests, an on-board Inertial Measurement Unit (IMU) was installed on the wing, providing synchronized measurements of 3D accelerations, angular rates, and attitude of the kite. The kite is steered by a single actuator, consisting in a linear motion system installed in the ground unit. The kite steering lines are attached to the opposite extremes of the moving carriage of the linear motion system, thus linking its position (regulated by an automatic flight control system) to the kite steering input. For more information on the prototype see e.g. [25], [26]. The UCSB prototype was used with three sizes of Leading-Edge-Inflated (LEI) tube kites in field experiments.

1) $6 m^{2}$ LEI tube kite: An example of filtering and estimation results for the smaller kite are shown in Figure 10. Observe in Figure 10a that the flight trajectory, although centered about $10^{\circ}$ to the right and slightly rotated counterclockwise, now follows more closely the lying-eight figure when compared to the UFSCkite experiment. As a consequence, cyclic oscillations for each completed orbit can be clearly seen not only in the magnitude of the lift (in Figure 10c and drag (in Figure 10d) forces, but also in the estimated wind at the kite position, in Figure $10 \mathrm{~b}$ Also note that the average lift force produced with this $6 \mathrm{~m}^{2}$ kite is around $600 \mathrm{~N}$, which is about twice as much in comparison to that obtained with the $3 \mathrm{~m}^{2}$ kite from the UFSCkite group. This seems reasonable if one considers roughly the same combined effect of average wind speed and coefficients of aerodynamic lift and equivalent efficiency in both cases (see model (20)).

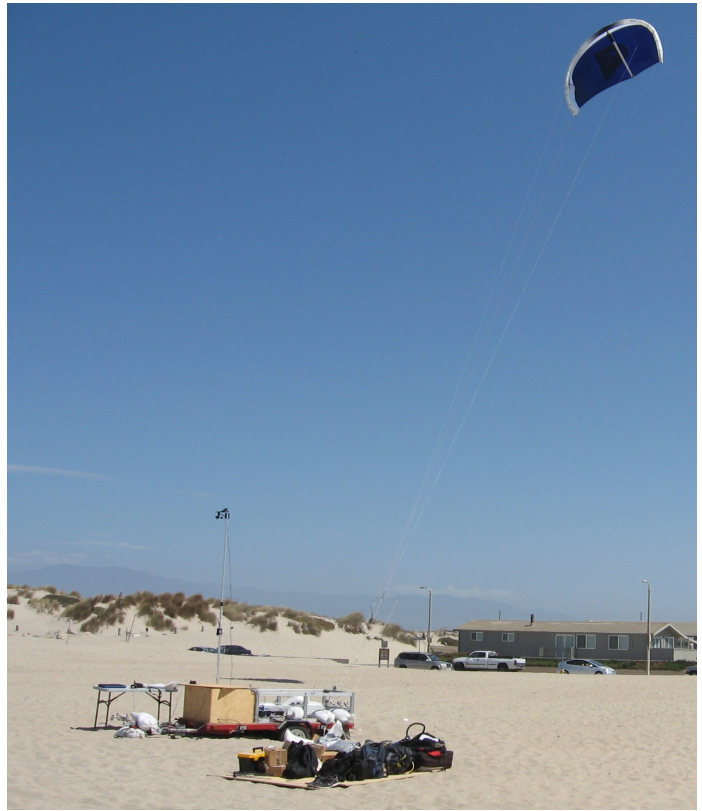

(a) Overview of the prototype in operation.

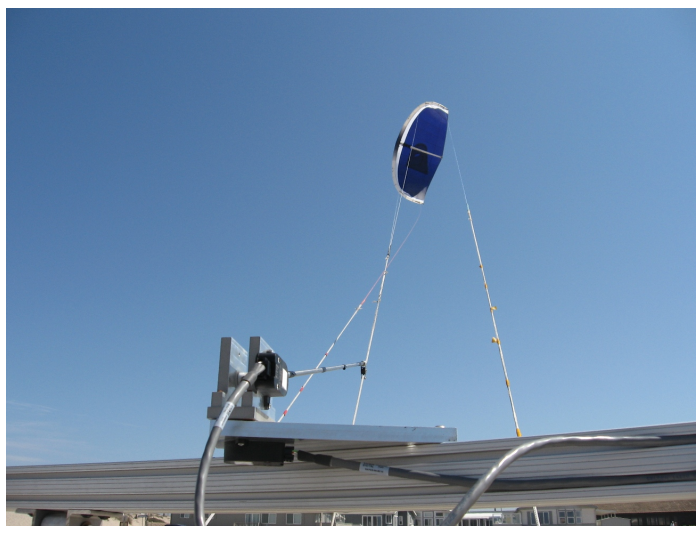

(b) Mechanism used for measuring the main tether angles.

Fig. 9: Small-scale prototype for the control of tethered wings built at the University of California, in Santa Barbara, USA.

Similarly to what was done with the UFSCkite prototype, to validate the results obtained from the field experiments with the UCSB prototype the EKF estimates were applied to model 20], yielding values of expected average traction force for each orbit which were compared to the average measured traction force for each orbit. The results are presented in Figure 11. In comparison to the $3 \mathrm{~m}^{2}$ kite, note how the points are now more concentrated around the line that represents a perfect match. This is likely due to the higher traction force obtained with the $6 \mathrm{~m}^{2}$ kite, keeping the main tether more taut and therefore reducing the modeling errors in the filter.

The identification of the aerodynamic lift coefficient curve $C_{L}(\Delta \alpha)$ is shown in Figure 12a Similarly to what was observed with the UFSCkite, the (partial) angle of attack oscillated in an interval of approximately $15^{\circ}$. However, note that the fitted curve, also obtained with a $3^{\text {rd }}$ degree polynomial, here comes close to 1.5 , which is quite high in comparison to the maximum lift coefficient of roughly 0.7 


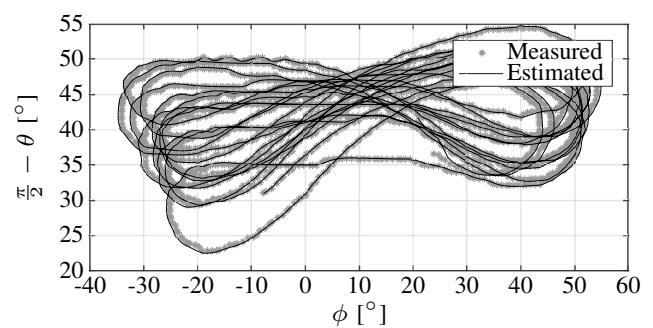

(a) Flight trajectory in the elevation-azimuth plane.

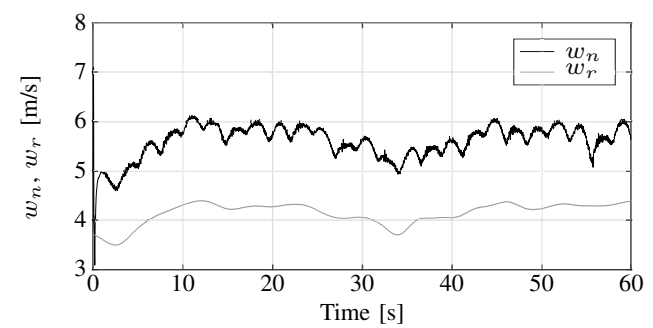

(b) Estimated wind at kite (black line) and reference (gray) height.

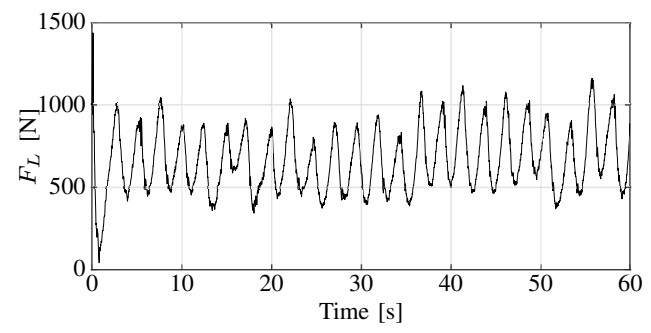

(c) Magnitude of the estimated lift force.

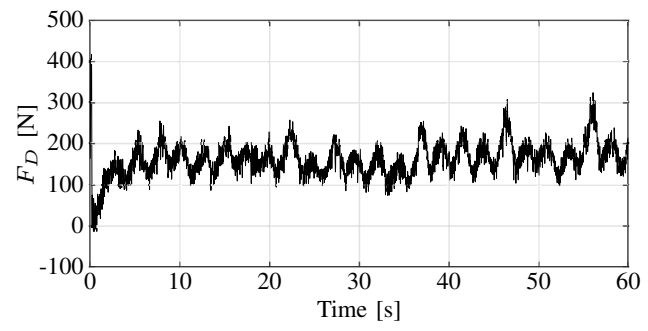

(d) Magnitude of the estimated equivalent drag force.

Fig. 10: Estimation results with the $6 \mathrm{~m}^{2}$ kite of the UCSB prototype during a field experiment.

achieved with the $3 \mathrm{~m}^{2}$ kite. Also note here that the dispersion of the estimated values of $C_{L}$ around the fitted curve is clearly smaller, something that could be expected in face of the better validation results shown in Figure 11. As for the identified equivalent efficiency, in Figure $12 \mathrm{~b}$, observe that it varies between 2 and 6, clearly in a lower interval compared to the variation between 3 and 8 in the case of the $3 \mathrm{~m}^{2}$ ram-air kite. This means that, despite the higher lift coefficient, the combination of tether drag of the UCSB prototype with the $6 \mathrm{~m}^{2}$ kite drag was greater than that of the UFSCkite prototype.

2) $9 \mathrm{~m}^{2}$ LEI tube kite: This was the LEI tube kite of intermediate size. Filtering results can be seen in Figure 13 Regarding the flight trajectory, observe in Figure 13a that it is now slightly rotated clockwise in contrast to what happened

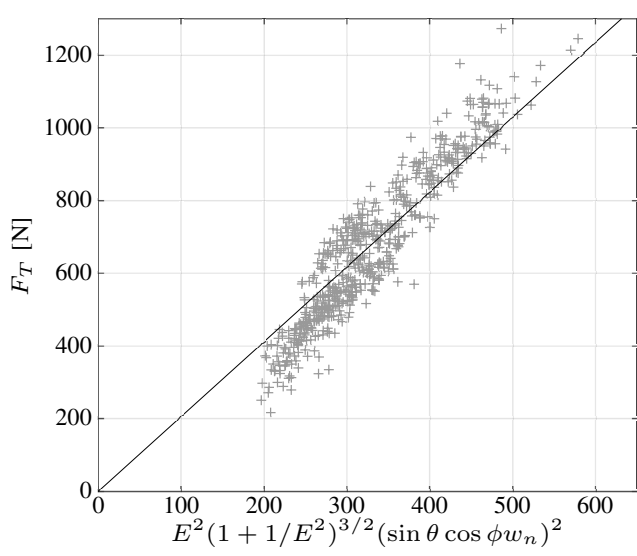

Fig. 11: Validation of the tether force estimates obtained in field tests with the UCSB prototype and a $6 \mathrm{~m}^{2}$ LEI tube kite.

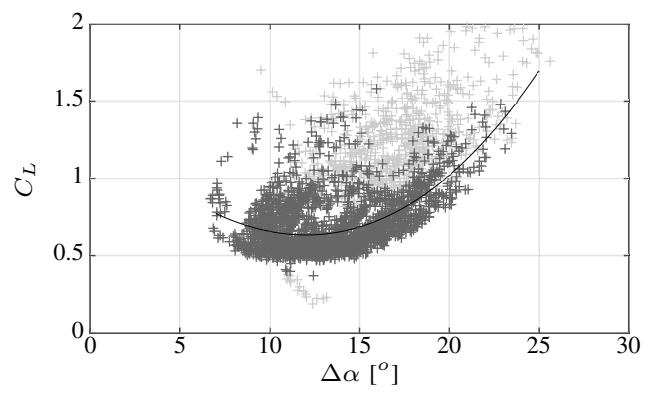

(a) Lift curve.

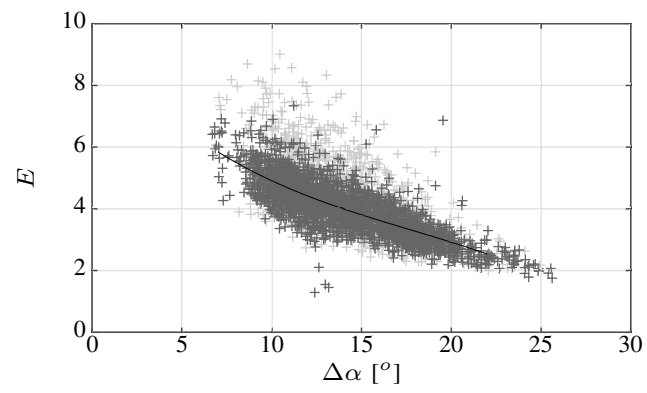

(b) Equivalent efficiency curve.

Fig. 12: Identification of the aerodynamic coefficients of the UCSB prototype with a $6 \mathrm{~m}^{2}$ LEI tube kite.

with the $6 \mathrm{~m}^{2}$ kite, although also following quite well the desired lying-eight pattern. In Figure 13b, it can be seen that the average wind at the kite was a little stronger than in the previous case, around $7 \mathrm{~m} / \mathrm{s}$. Due to the $50 \%$ larger area and the slightly stronger wind than in the case of the $6 \mathrm{~m}^{2}$ kite, the magnitude of the lift force in Figure $13 \mathrm{c}$ now presents a larger amplitude of oscillation, roughly between $1 \mathrm{kN}$ and $2.5 \mathrm{kN}$, centered around $1.7 \mathrm{kN}$, which is about twice the average lift force of the $6 \mathrm{~m}^{2}$ kite. Also as one could have expected, the magnitude of the drag force, shown in Figure $13 \mathrm{~d}$, increased due to the stronger wind and consequently faster kite and larger apparent wind speed.

Once again, the same validation procedure of the previous 


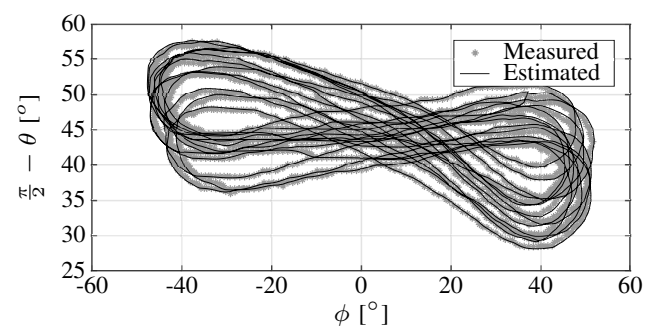

(a) Flight trajectory in the elevation-azimuth plane.

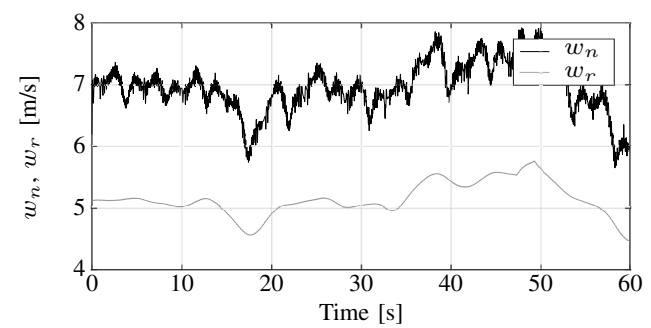

(b) Estimated wind at kite (black line) and reference (gray) height.

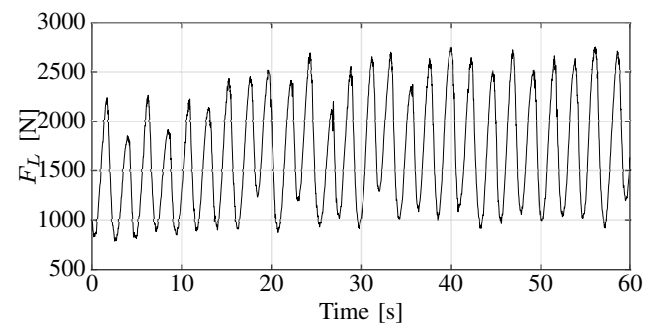

(c) Magnitude of the estimated lift force.

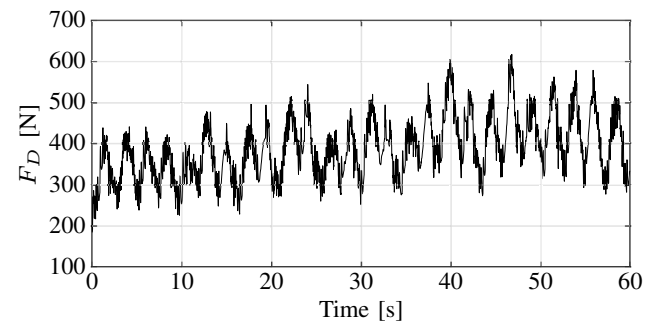

(d) Magnitude of the estimated equivalent drag force.

Fig. 13: Estimation results with the $9 \mathrm{~m}^{2}$ kite of the UCSB prototype during a field experiment.

experiments was applied. The results can be seen in Figure 14 Note that the dispersion of the points around the theoretical perfect match represented by the straight line is now even lower than in the case of the $6 \mathrm{~m}^{2}$ kite. This was expected, as the tether traction force is significantly high, allowing the tethers to be more taut and therefore causing model (20) to be more representative of the real system average behavior, as already discussed.

As for the estimated aerodynamic coefficients, observe in Figure 15a how the fitted lift curve, $C_{L}(\Delta \alpha)$, now features a clear lower inflexion point, at $\Delta \alpha \approx 13^{\circ}$. This is quite a different behavior than that of the lift curve assumed for the simulations (see Figure $5 \mathrm{~b}$ ). In the case of the $9 \mathrm{~m}^{2}$ kite, the observed behavior is that, if the kite is operating at $\Delta \alpha \approx 5^{\circ}$

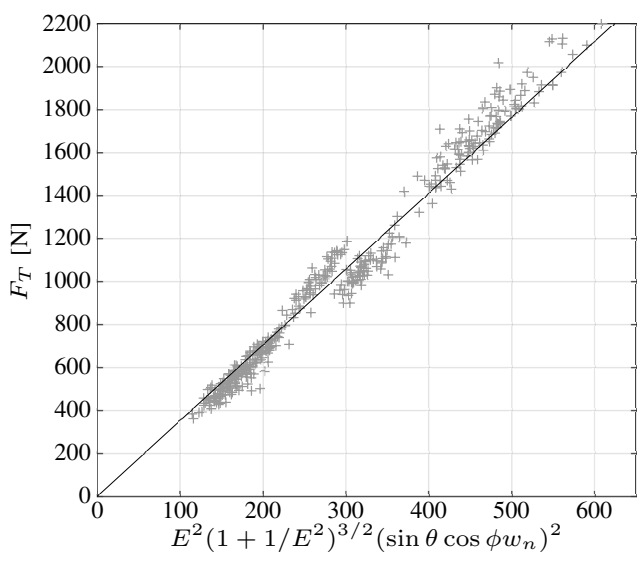

Fig. 14: Validation of the estimates obtained in a field test with the UCSB prototype and a $9 \mathrm{~m}^{2}$ LEI tube kite.

and the angle of attack starts increasing, first the lift coefficient (and force) decreases to a minimum before it can start growing. This may be due to the flexible structure of the LEI tube kite wing, a behavior that could be an interesting topic of study by aerodynamic engineers and kite manufacturers. Regarding the aerodynamic efficiency, in Figure $15 \mathrm{~b}$, the fitted curve is very similar to that obtained for the $3 \mathrm{~m}^{2}$ foil kite and the $6 \mathrm{~m}^{2}$ LEI tube kite, except that the dispersion of the estimated points is now even lower.

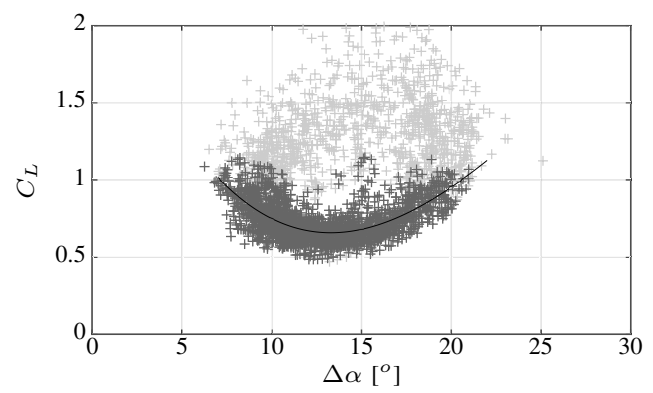

(a)

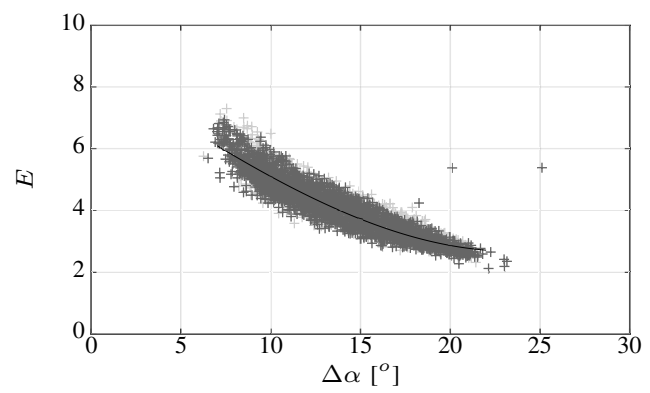

(b)

Fig. 15: Identification of the aerodynamic coefficients of the UCSB prototype with a $9 \mathrm{~m}^{2}$ LEI tube kite.

Another interesting result from the filter outputs is shown for the $9 \mathrm{~m}^{2}$ LEI tube kite in Figure 16, where the estimated equivalent aerodynamic efficiency $E$ is plotted against the steering input $u_{s}$. Both of these quantities vary in a cyclic- 
fashion throughout a lemniscate-type orbit, depicted in Figure 13a. For instance, as the kite goes down the lemniscate through the trajectory center, it speeds up approximately in a straight line, hence $u_{s}$ can be very low in magnitude whereas $E$ increases because of the decrease in the angle of attack through the $\Delta \alpha$ share. But then, in order to execute an U-turn by climbing up, a strong steering input is applied. Recall that when this large input is applied the speed (and efficiency) are near their maximum values and the kite takes a certain time to respond to this input. With the kite response, the speed decreases with the U-turn due to drag and gravity effects, leading $\Delta \alpha$ to increase and $E$ to decrease. This fact suggests there are several points in the trajectory, near the bottom parts of the lemniscate-like figure, with large input and large efficiency values. The other half orbit would have a similar behavior of what has been just described, except that the steering command would have the opposite direction. At the end of an orbit a symmetric trajectory of the evolution in time of the $E\left(u_{s}\right)$ curve should be obtained, a behavior that indeed is observed in Figure 16

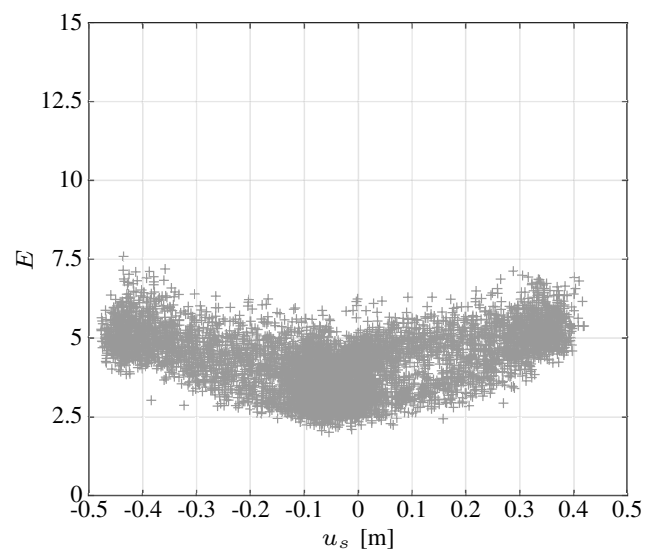

Fig. 16: Estimated aerodynamic efficiency versus the steering command for the UCSB prototype with a $9 \mathrm{~m}^{2}$ LEI tube kite.

3) $12 m^{2}$ LEI tube kite: The last experimental results refer to a $12 \mathrm{~m}^{2}$ LEI tube kite, the largest among the four kites considered in this paper. In Fig. 17a it is clear that the flight trajectory in this case is a well-shaped lemniscate centered around $\phi=0^{\circ}$, quite symmetric and horizontally aligned (i.e., not tilted). A difference that stands out from the previous experiments is that the estimated wind speed at the kite height, plotted in Figure 17b is significantly lower, between $3.5 \mathrm{~m} / \mathrm{s}$ and $4 \mathrm{~m} / \mathrm{s}$. Hence, the increase of $33 \%$ in the kite area was not enough to compensate for the decrease in the wind speed. As a consequence, the magnitude of the lift force $F_{L}$ (which has the largest contribution to $F_{T}$ ) oscillated with a mean value around $1 \mathrm{kN}$, clearly lower than the average traction force of the $9 \mathrm{~m}^{2}$ kite. Because of the lower wind, the magnitude of the drag force, in Figure 17d, also turned out to be lower in comparison to the previous kite experiment.

Figure 18 contains the validation results of the EKF estimates for the $12 \mathrm{~m}^{2}$ kite. It is interesting how the dispersion of the points around the perfect match line is apparently the lowest in comparison to the other experiments, despite the low

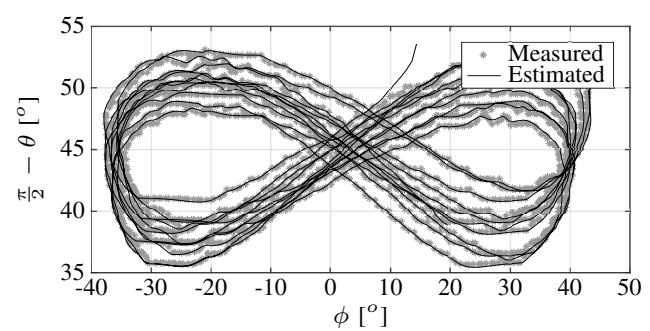

(a) Flight trajectory in the elevation-azimuth plane.

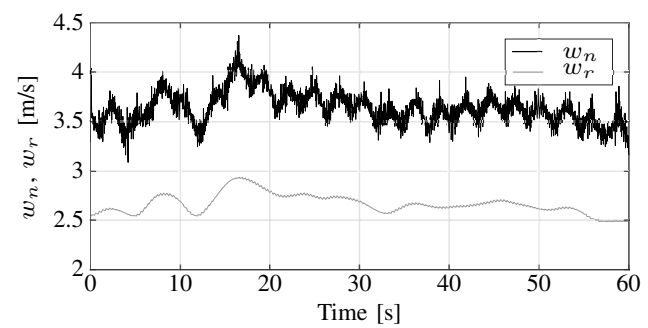

(b) Estimated wind at kite (black line) and reference (gray) height.

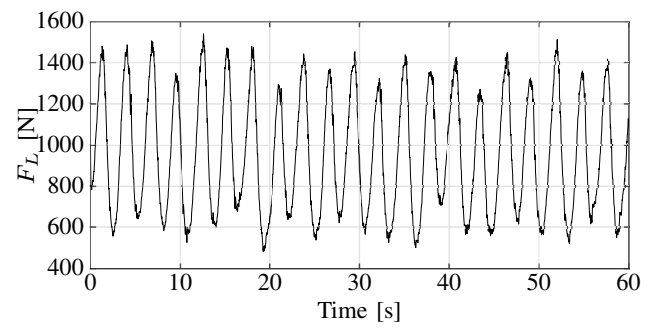

(c) Magnitude of the estimated lift force.

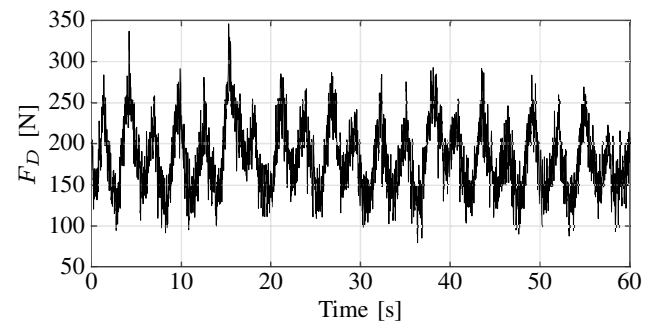

(d) Magnitude of the estimated equivalent drag force.

Fig. 17: Estimation results with the $12 \mathrm{~m}^{2}$ kite of the UCSB prototype during a field experiment.

wind speed and therefore larger modeling errors, as already discussed. This low dispersion is probably due to the well behaved flight trajectory, which followed a standard lemniscate shape with only relatively minor deviations from orbit to orbit, yielding average values for the estimates that did not vary so much with time.

The estimated aerodynamic lift curve is shown in Figure 19a. Note that the concavity of the fitted curve is the largest among all kites investigated in this paper. Again, this may be caused by a design feature of the wing, which in this case is accentuated by the larger size. As for the aerodynamic efficiency, presented in Figure 19b, it seems to vary almost linearly with the angle of attack.

The behavior of the estimated aerodynamic efficiency 


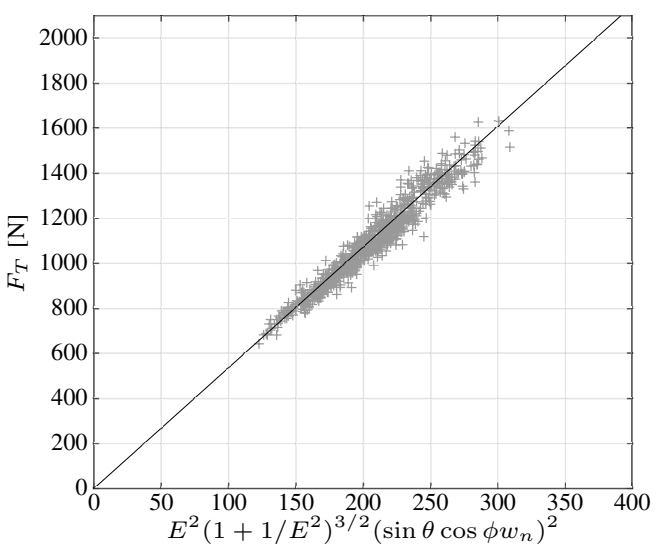

Fig. 18: Validation of the estimates obtained in a field test with the UCSB prototype and a $12 \mathrm{~m}^{2}$ LEI tube kite.

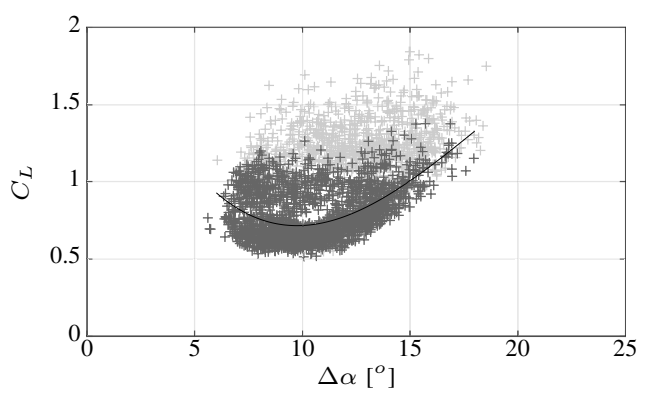

(a)

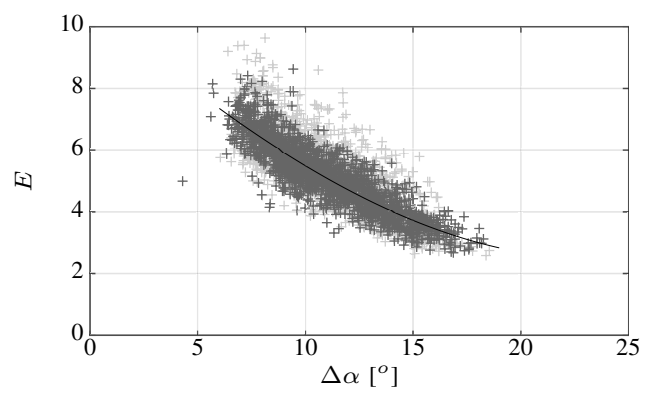

(b)

Fig. 19: Identification of the aerodynamic coefficients of the UCSB prototype with a $12 \mathrm{~m}^{2}$ LEI tube kite.

against the steering input for this kite is shown in Figure 20 In this case the plot is asymmetric: the efficiency values for strong positive steering inputs are higher than those for similarly strong but negative steering inputs. This is probably due to a repair that was executed on one of the kite tips during the experimental campaign. However, even if the kite were perfectly balanced, asymmetries in the $E\left(u_{s}\right)$ plot could have other causes, such as a possible misalignment between the center of the trajectory and the prevalent wind direction (i.e. the true $\phi=0^{\circ}$ coordinate), or asymmetric bridle lines. Hence, the derived estimates could be used to adjust the trajectory to improve crosswind operation and to detect in-flight changes of the kite for predictive maintenance strategies.

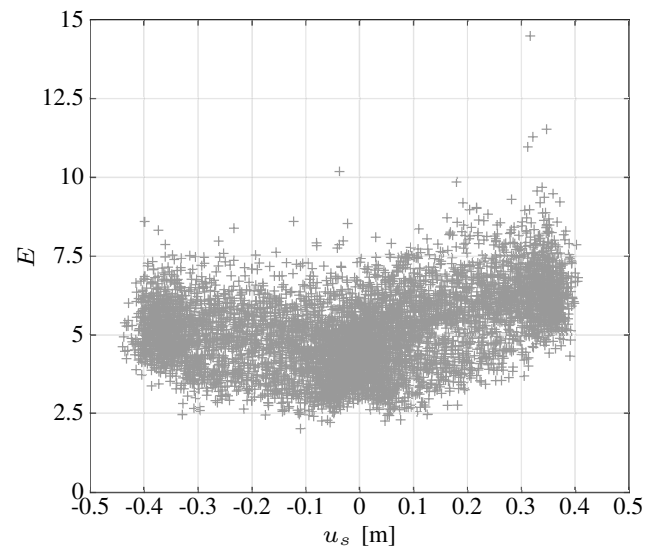

Fig. 20: Estimated aerodynamic efficiency versus the steering command for the UCSB prototype with a $12 \mathrm{~m}^{2}$ LEI tube kite.

\section{FINAL REMARKS}

This paper presented a state and parameter estimation strategy based on an Extended Kalman Filter (EKF) formulation and on an embedded model of the flight dynamics in natural coordinates. Its main contributions include the enhancement of the wing flight dynamics model with respect to a preliminary work presented in [16]; the demonstration of the effectiveness of an orthogonality constraint enforced in the filtering stage of the EKF, and the identification of the aerodynamic lift and equivalent efficiency curves of four different flexible wings with extensive experimental data, thus demonstrating the potential of the algorithm. The latter contribution is specially relevant for applications involving flexible wings because the characteristics of the kite can be assessed during normal operation and without the need of cumbersome wind tunnel experiments. The filter is also capable of estimating important quantities such as the kite position, velocity and acceleration, and the wind conditions at flight altitude, which might be interesting for the evaluation of potential installation sites.

There are several promising directions to pursue as future works based on the results presented in this paper. By monitoring the estimated aerodynamic efficiency (which accounts for both wing drag and tether drag), it could be possible to detect in-flight anomalies such as asymmetries in the kite's canopy and small ruptures or degradation of its fabric. This information could be valuable when deciding whether kite maintenance is required. As a matter of fact, the estimated curves of aerodynamic equivalent efficiency turned out to differ significantly from what one would have expected from the literature, presenting a lower inflection point at a low angle of attack. This may be a structural feature of the wing, possibly because of its flexibility, and is an interesting topic for further investigation.

Another interesting point for investigation is the possibility of further enhancing the accuracy of the estimates by making use of other sources of information such as ranging devices, camera systems, or inertial units. The inclusion of direct measurements of the apparent wind, obtained e.g. from a small wind turbine or Pitot tube mounted on the wing, is also an 
option that deserves attention, given that it could considerably improve the wind estimates and therefore mitigate the uncertainty in the estimated aerodynamic lift curves.

As for the filtering strategy itself, even though the results obtained from the simulation and the field tests suggest a good convergence property of the estimates, it is well-known that the EKF does not offer any theoretical guarantee of convergence. This is a limitation that could be overcome through the application of more sophisticated estimation methods such as moving-horizon estimators (MHE).

Finally, it was also pointed out how eventual horizontal or vertical asymmetries in the scatter plot of the estimated equivalent aerodynamic efficiency versus the steering input could be explored to give us important information. By further investigating the root causes behind these asymmetries, it could be possible to identify misalignments between the center of the flight trajectory and the prevalent wind direction, or even infer asymmetries in the kite itself (e.g. induced by material wear).

\section{REFERENCES}

[1] U. Ahrens, M. Diehl, and R. Schmehl, Eds., Airborne Wind Energy. Berlin Heidelberg: Springer, 2013.

[2] A. Cherubini, A. Papini, R. Vertechy, and M. Fontana, "Airborne wind energy systems: A review of the technologies," Renewable and Sustainable Energy Reviews, vol. 51, pp. 1461-1476, July 2015.

[3] B. Houska and M. Diehl, "Optimal control for power generating kites," in Proceedings of the 9th European Control Conference. Kos, Greece: IEEE, 2007, pp. 3560-3567. [Online]. Available: http://ieeexplore.ieee.org/stamp/stamp.jsp?arnumber=7068861

[4] L. Fagiano, "Control of tethered airfoils for high-altitude wind energy generation," Ph.D. dissertation, Politecnico di Torino, 2009. [Online]. Available: http://hdl.handle.net/11311/1006424

[5] P. Williams, B. Lansdorp, R. Ruiterkamp, and W. Ockels, "Modeling, simulation, and testing of surf kites for power generation," in Proceed ings of the AIAA Modeling and Simulation Technologies Conference and Exhibit, Honolulu, HI, USA, 2008, paper AIAA Paper 2008-6693.

[6] M. Canale, L. Fagiano, and M. Milanese, "High altitude wind energy generation using controlled power kites," IEEE Transactions on Control Systems Technology, vol. 18, no. 2, pp. 279 -293, mar. 2010.

[7] J. H. Baayen and W. J. Ockels, "Tracking control with adaption of kites," IET Control Theory and Applications, vol. 6, no. 2, pp. 182-191, 2012.

[8] C. Jehle and R. Schmehl, "Applied tracking control for kite power systems," AIAA Journal of Guidance, Control, and Dynamics, vol. 37 , no. 4, pp. 1211-1222, 2014.

[9] L. Fagiano, A. U. Zgraggen, M. Morari, and M. Khammash, "Automatic crosswind flight of tethered wings for airborne wind energy: Modeling, control design and experimental results," IEEE Transactions on Control Systems Technology, vol. 22, no. 4, pp. 1433-1447, July 2013.

[10] M. Erhard and H. Strauch, "Flight control of tethered kites in autonomous pumping cycles for airborne wind energy," Control Engineering Practice, vol. 40, no. 0, pp. 13-26, 2015.

[11] A. U. Zgraggen, L. Fagiano, and M. Morari, "Automatic retraction and full-cycle operation for a class of airborne wind energy generators," IEEE Transactions on Control Systems Technology, vol. 24, no. 2, pp. 594-608, March 2016.

[12] O. H. Hjuske, "State estimation and kalman filtering of tethered airfoils," Master's thesis, Norwegian University of Science and Technology, 2011. [Online]. Available: https://brage.bibsys.no/xmlui/bitstream/handle/ 11250/260392/437114_FULLTEXT01.pdf?sequence=1\&isAllowed=y

[13] M. Ranneberg, "Sensor setups for state and wind estimation for airborne wind energy converters," Cornell University Library, September 2013.

[14] T. A. Wood, H. Hesse, A. U. Zgraggen, and R. S. Smith, "Modelbased identification and control of the velocity vector orientation for autonomous kites," in 2015 American Control Conference (ACC). Chicago, IL: IEEE, July 2015, pp. 2377-2382.

[15] R. Saraiva, M. De Lellis, E. Schmidt, and A. Trofino, "Dynamics identification, filtering and control design for power kites," in Proceedings of the 20th IFAC World Congress, ser. 1, vol. 50. Toulouse, France: IFAC, July 2017, pp. 11938-11943.
[16] E. Schmidt, M. De Lellis, R. Saraiva, and A. Trofino, "State estimation of a tethered airfoil for monitoring, control and optimization," in Proceedings of the 20th IFAC World Congress, ser. 1, vol. 50. Toulouse, France: IFAC, July 2017, pp. 13246-13251.

[17] M. Erhard and H. Strauch, "Control of towing kites for seagoing vessels," IEEE Transactions on Control Systems Technology, vol. 21, no. 5, pp. 1629-1640, September 2013.

[18] S. Gros and M. Diehl, "Modeling of airborne wind energy systems in natural coordinates," in Airborne Wind Energy, U. Ahrens, M. Diehl, and R. Schmehl, Eds. Berlin Heidelberg: Springer, 2013, ch. 10, pp. 181-203.

[19] M. De Lellis, "Airborne wind energy with tethered wings: Modeling, analysis and control," Ph.D. dissertation, Federal University of Santa Catarina, Florianopolis (SC), Brazil, October 2016, (Marcelo De Lellis Costa de Oliveira). [Online]. Available: http://www.bu.ufsc.br/teses/ PEAS0222-T.pdf

[20] C. L. Archer, "An introduction to meteorology for airborne wind energy," in Airborne Wind Energy, ser. Green Energy and Technology, U. Ahrens, M. Diehl, and R. Schmehl, Eds. Berlin Heidelberg: Springer-Verlag, 2014, ch. 5, pp. 81-94.

[21] D. Simon, "Kalman filtering with state constraints: a survey of linear and nonlinear algorithms," IET Control Theory and Applications, vol. 4, no. 8, pp. 1303-1318, 2010.

[22] S. Haykin, Ed., Kalman Filtering and Neural Networks. John Wiley \& Sons, 2001

[23] M. De Lellis, R. Saraiva, and A. Trofino, "Turning angle control of power kites for wind energy," in Proceedings of the IEEE 52nd Annual Conference on Decision and Control. Firenze, Italy: IEEE, December 2013, pp. 3493-3498.

[24] U. Fechner, R. van der Vlugt, E. Schreuder, and R. Schmehl, "Dynamic model of a pumping kite power system," Renewable Energy, vol. 83, pp. 705-716, November 2015.

[25] L. Fagiano, K. Huynh, B. Bamieh, and M. Khammash, "On sensor fusion for airborne wind energy systems," IEEE Transactions on Control Systems Technology, vol. 22, no. 3, pp. 930-943, May 2014.

[26] L. Fagiano and T. Marks, "Design of a small-scale prototype for research in airborne wind energy," IEEE/ASME Transactions on Mechatronics, vol. 20, no. 1, pp. 166-177, February 2015. 